\title{
Strangeness production in a constituent quark model
}

\author{
F.Becattini and G.Pettini* \\ Department of Physics, University of Florence and I.N.F.N., Florence, Italy \\ E-mail: becattini@fi.infn.it, pettini@fi.infn.it
}

\begin{abstract}
We develop a model to calculate strangeness production in both elementary and heavy ion collisions, within the framework of a statistical approach to hadronisation. Calculations are based on the canonical partition function of the thermal NambuJona-Lasinio model with exact conservation of flavor and color. It turns out that the growth of strange quarks production in heavy ion collisions is due to the initial excess of non-strange matter over antimatter, whereas a suppression occurs for elementary collisions, owing to the constraint of exact quantum charges conservation over small volumes.
\end{abstract}

\section{The Statistical Model of Hadronisation (SHM) and the Wroblewski factor $\lambda_{S}$}

A statistical calculation of hadron multiplicities in high energy collisions has been developed in ref. The SHM relies on the assumption of equiprobability of multi-hadronic states originating from hadronising clusters, entailing that particle production in hadronisation of both elementary (EC) and heavy ion (HIC) collisions can be treated as an equilibrium process. The main advantage of the model is the low number of free parameters necessary to reproduce the observed hadron multiplicities. These are: the total volume $V$ of the set of clusters, temperature $T$, and a phenomenological parameter $\gamma_{S}$ reducing the production of strange particles with respect to a fully chemically equilibrated hadron gas. The exact conservation of initial electric, baryon and strange total quantum charges is enforced in the 
model. However, when dealing with HIC, the conservation of the initial baryon number can be imposed only on the average and the baryon chemical potential $\mu_{B}$ has to be introduced. A remarkable outcome of the SHM is that the temperature fitted in any EC is almost constant $(T \sim 160 \mathrm{MeV})$, and very close to that obtained in HIC with small fitted $\mu_{B}$. Furthermore, for different HIC, to an increase of the fitted value of $\mu_{B}$ corresponds a decrease of the value of $T$.

An interesting insight in strangeness production is obtained when studying the so-called Wroblewski factor, the ratio between newly produced valence strange and light quark pairs $\lambda_{S}=2\langle s \bar{s}\rangle /(\langle u \bar{u}\rangle+\langle d \bar{d}\rangle)$, which is fairly constant in several EC, with $\sqrt{s}$ spanning two orders of magnitude, whereas it is significantly higher and more variable in $\mathrm{HIC}$ (

The peculiar behaviour of $\lambda_{S}$, together with the apparent universality of the scale of temperature fitted within EC, suggest to look for a more fundamental description of strangeness production, by employing effective models (EM) having quarks as fundamental degrees of freedom.

\section{Connection with effective models}

In using EM, the same physical scheme of the SHM is kept. However, we now assume the formation of a set of hadronising clusters endowed with given $U, D, S$ and color charges, in which every allowed quantum state is equally likely. The assumption of suitable maximum disorder fluctuations of cluster flavor charges is retained 1 , which allows to impose exact flavor conservation over the total volume $V$. Further conjectures are introduced:

1. Temperature $T$ and baryon chemical potential $\mu_{B}$, fitted within the SHM, are interpreted as the critical values for deconfinement and (approximate) chiral symmetry restoration

2. The produced s quarks, or at least the ratio $s / u$, survive in the hadronic phase

3. Each single cluster is a color singlet 
The first assumption is the strongest one, as it implies that hadronisation itself is assumed to be a critical process. The aforementioned constancy of fitted temperature for many collisions 2 and the consistency with other estimates, support this identification. Assumption (2) implies that full chemical equilibrium is assumed at constituent quark level. Thus, no parameter such as $\gamma_{S}$ is now required to account for strangeness production. Conversely, assumption (3) introduces a further parameter, a characteristic cluster size $V_{c}$ over which color is exactly conserved, which, together with the constancy of $T$ and a weak dependence of $\lambda_{S}$ itself on the total volume $V$, contributes in stabilizing $\lambda_{S}$ for EC. On the other hand, a large $V_{c}$ in HIC could be a signal for color deconfinement over large volumes.

The simplest effective model to start with is the thermal Nambu-Jona-Lasinio model $\mathrm{B}$. It cannot account for deconfinement but it does embody chiral symmetry breaking and its restoration $(\chi \mathrm{SR})$, which is expected to occur at the same critical point $\mathrm{\theta}$. Although not renormalizable, the NJL model shares the main features with other effective models, referred in literature as ladder-QCD $\mathrm{O}$. Actually the phase diagram for $\chi \mathrm{SR}$ exhibits a tricritical point in the chiral limit, separating second order from first order phase transitions. We are interested to include the current quark masses, in which case a smooth cross-over transition is expected for the light quarks in the second order region (in which we are interested in the present work). This implies a quasi-critical behavior of the light quark condensates and a smooth decrease of the strange one for higher temperatures. The same happens to the constituent quark masses which are the physical quantities, together with the UV cutoff $\Lambda$ B, affecting the number of quarks (or antiquarks). Actually, the thermodynamics of the mean-field NJL model can be derived by a free Dirac Hamiltonian having constituent quark masses replacing the current ones. The dependence of quark multiplicities on $V, V_{c}$ emerges when requiring exact conservation of quantum flavor and color charges by restricting the partition function to the allowed set of multi-particle states. This is accomplished by means of standard methods 6 and implies a five-dimensional integration over the group $S U(3)_{c} \times U(1)_{u} \times U(1)_{d} \times U(1)_{s}$. Calculations are shown in detail in ref. $\mathrm{Z}$. 


\section{Results}

\section{A. Heavy Ions}

For HIC, the fitted total volumes $\mathrm{\theta}$ are large enough to disregard the fluctuations of flavor quantum numbers. Then, if $V_{c}$ is large enough, it can be explicitly checked that a grandcanonical calculation of quark multiplicities is reliable. This implies that $\lambda_{S}$ can be obtained by the simple grand-canonical formula for a gas of free constituent quarks with an UV cutoff limiting the momentum integration 3 , and it is independent on $V, V_{c}$. Temperature $T$ and chemical potentials $\mu_{i}$ are taken coincident with those fitted within the SHM. A first comparison with the SHM predictions can be performed by considering the values of the constituent quark masses $M_{i}$ for the strange quark $\left(\mu_{s}=0\right)$ and for a light quark $q$ $\left(\mu_{q} \sim \mu_{B} / 3\right)$ with mass $\hat{m}=\left(m_{u}+m_{d}\right) / 2$, as free parameters to be determined imposing the SHM value of $\lambda_{S}$ for a given process. A more complete analysis can be obtained by using the thermal NJL model in a predictive way. The coincidence of the cross-over point with the best fit of the SHM is possible by tuning the parameter $T_{0}$, defined in ref 3 , which controls the strength of the six-fermion term and which physically represents the critical temperature for $U(1)_{A}$ restoration. Then the cross-over line, obtained by studying the chiral light quark susceptibility can be compared with the fitted values of $T$ and $\mu_{B}$ in the SHM, as well as $\lambda_{S}$ along the critical line. In this case the constituent masses $M_{i}\left(T, \mu_{i}\right)$ are predicted by minimizing the NJL grand canonical potential. A good agreement is found with $T_{0}=170$ $\mathrm{MeV}$ for various HIC.

However, as it turns out that $M_{s}$ is almost independent on the choice of $T_{0}$, the model needs the specification of only one parameter, either $T_{0}$ or $M_{q}$. This is shown in Fig. 2, which shows, on the other hand, that under the reasonable assumption that the light quark

constituent mass has sensibly decreased at the cross-over point, the agreement with the SHM is guaranteed by $M_{s}(T=0) \sim 500 \mathrm{MeV}$. The limit $\Lambda \rightarrow \infty$ serves to show that the UV contribution is not expected to change this picture, even if the analysis in renormalizable 
models such as ladder-QCD 目 is necessary.

\section{B. Elementary Collisions}

The location of the cross-over point for EC, $T \sim 160 \mathrm{MeV}\left(\mu_{B}=0\right)$, as well as $M_{i}$, are obtained in the predictive NJL model with $T_{0}=170 \mathrm{MeV}$ in the infinite volume limit. Consequently, quark multiplicities depend on the volume only through the explicit dependence of the canonical partition function on $V, V_{c}$. This approximation already allows us to observe canonical suppression. We find consistency with our physical picture, as far as processes with zero initial electric charge are concerned, whereas for pp collisions the Pauli exclusion principle and canonical suppression balance each other and $\lambda_{S}$ remains constant around $\sim 0.3$. In Fig. 3 we plot the predictions of the model compared to the SHM expectations for $\mathrm{e}^{+} \mathrm{e}^{-}$collisions.

\section{Conclusions}

We have studied strangeness production within a statistical model of hadronisation by using an effective model with constituent quarks. The study supports the idea that the SHM analysis may help in effective models building for the QCD phase transition, whereas EM may give a natural explanation of strangeness suppression in EC, assuming full chemical equilibrium, as due to the absence of baryon chemical potential and to canonical suppression working in small volumes. A significative discrepancy is found only in pp collisions, where further analysis is required. 


\section{REFERENCES}

1. F. Becattini, Z. Phys. C69 485 (1996); F. Becattini and U. Heinz, Z. Phys. C76 269 (1997).

2. F. Becattini, in Strange Quarks in Matter 2001, hep-ph 0202071, to appear in J. Phys. $\mathbf{G}$, and references therein.

3. T. Hatsuda and T. Kunihiro, Phys. Rep. 247, 221 (1994).

4. F. Karsch and E. Laermann, Phys. Rev. D59 031501 (1999).

5. A. Barducci et al., Phys. Rev. D41, 1610 (1990); A. Barducci et al., Phys. Rev. D49, 426 (1994) and references therein.

6. K. Redlich and L. Turko, Z. Phys. C5, 201 (1980).

7. F. Becattini and G. Pettini in QCD@Work, AIP PROCEEDINGS, Vol. 62, 333 (2001); F. Becattini and G. Pettini, in preparation. 


\section{FIGURES}

Fig. 1. $\lambda_{S}$ as a function of $\sqrt{s}$ in several kinds of collisions (from ref. 2).

Fig. 2. Constituent quark masses $M_{q}, M_{s}$ determined by $\lambda_{S} \simeq 0.45$ as fitted in $\mathrm{Pb}-\mathrm{Pb}$ collisions at $\sqrt{s}_{N N}=17.4 \mathrm{GeV}$. The hatched lighter region corresponds to the free constituent quark gas with $\Lambda=631 \mathrm{MeV}$ as in ref. $3^{3}$ whereas the darker hatched region is for $\Lambda=\infty$. Together, we plot the predictions of the NJL model with $T_{0}$ ranging from zero (no KMT term) to $\infty$ from left to right (solid line). The black point is for $T_{0}=170 \mathrm{MeV}$. The dashed pointed horizontal and vertical lines correspond to the $T=\mu_{B}=0$ constituent masses values for the strange quark and for the light quark $q$ respectively.

Fig. 3. Calculated $\lambda_{S}$ in $\mathrm{e}^{+} \mathrm{e}^{-}$collisions at $T=160 \mathrm{MeV}$ within the NJL model ${ }^{3}$ with $T_{0}=170$ $\mathrm{MeV}$, as a function of the total volume $V$ for various single cluster volumes $V_{c}$. The dotted line is with $V_{c}=V$ and the arrow indicates the grand-canonical limit. The horizontal bands are the ranges of fitted $\lambda_{S}$ values in the SHM (see Fig. 1). 


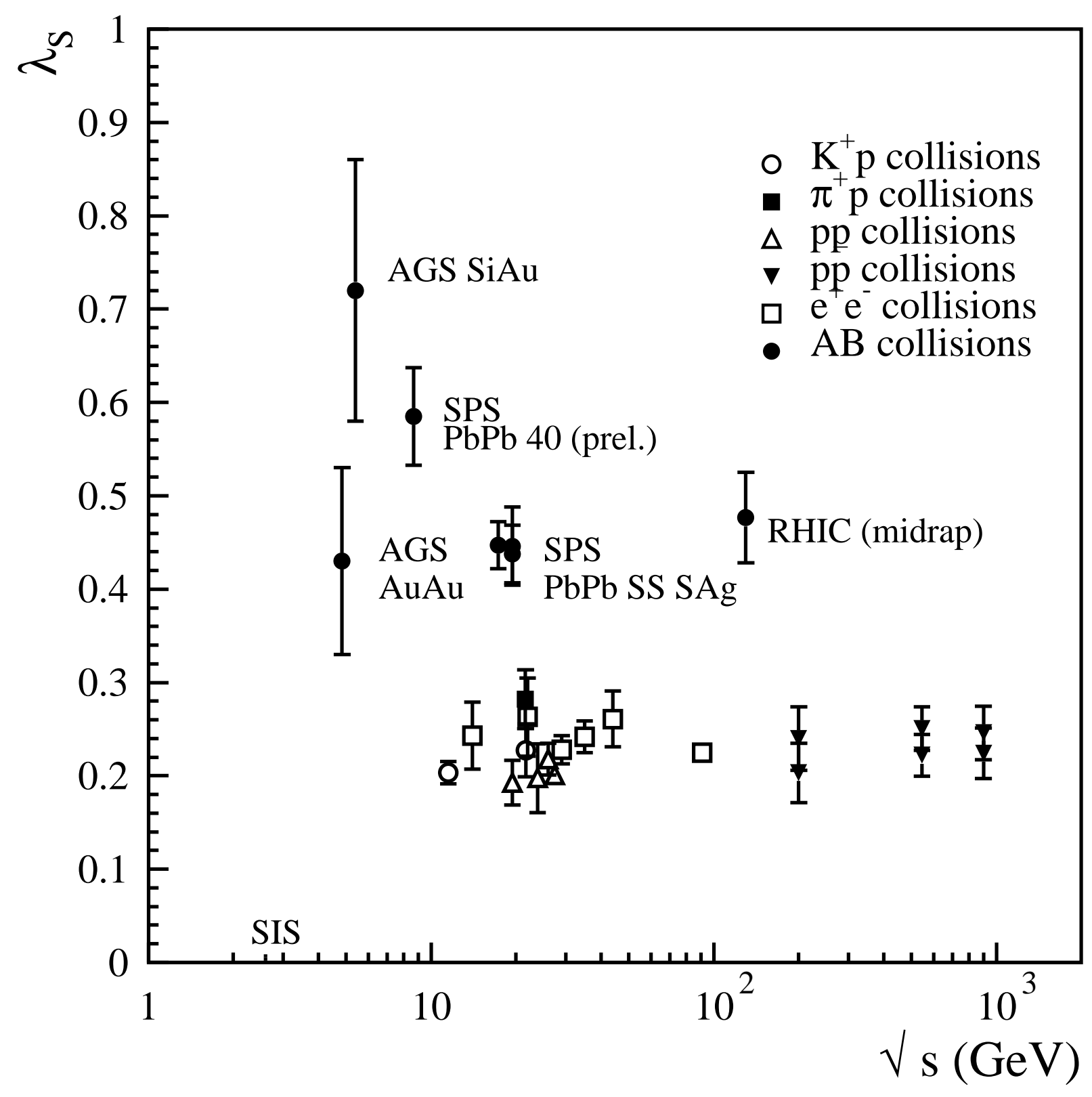




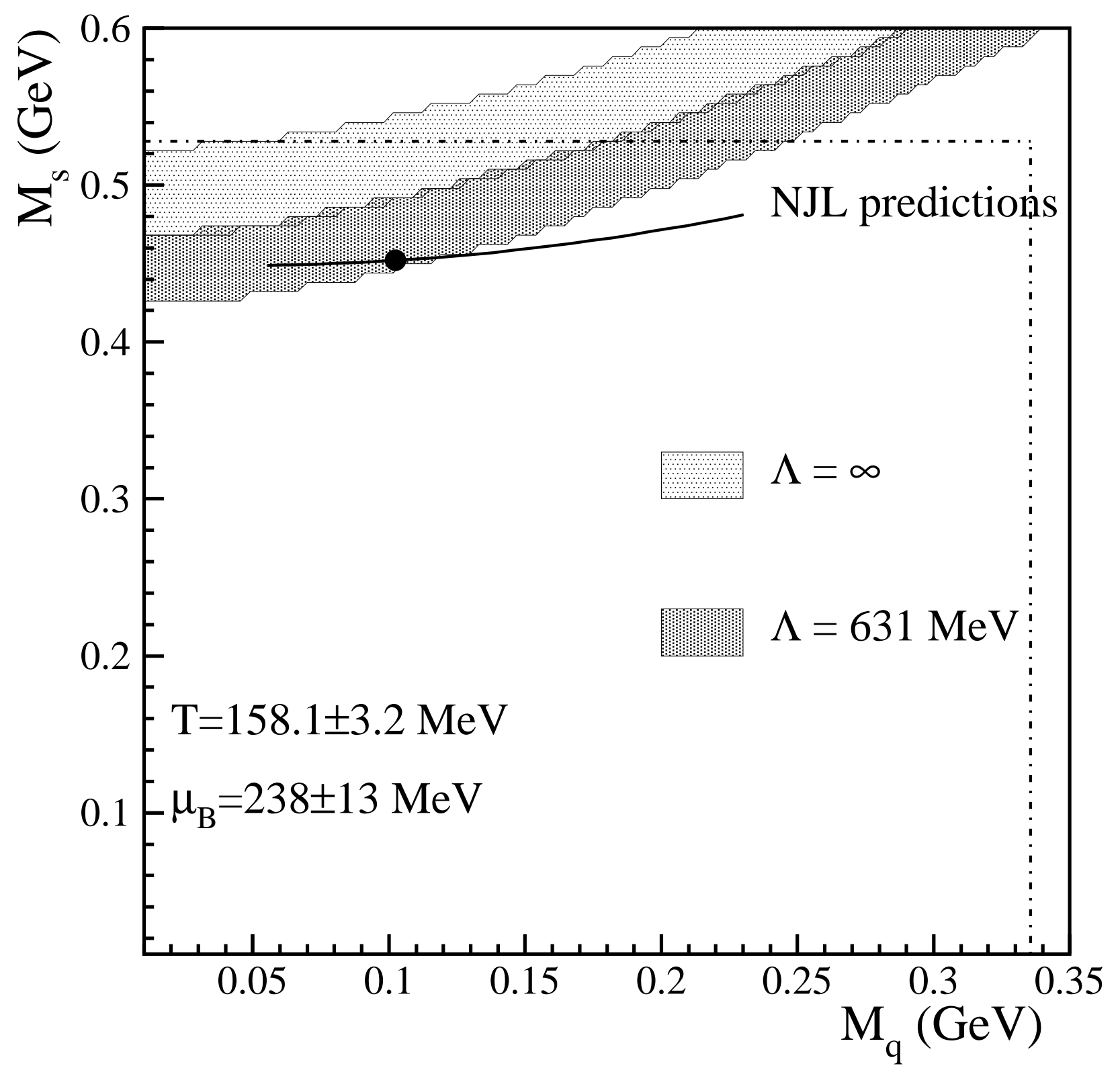




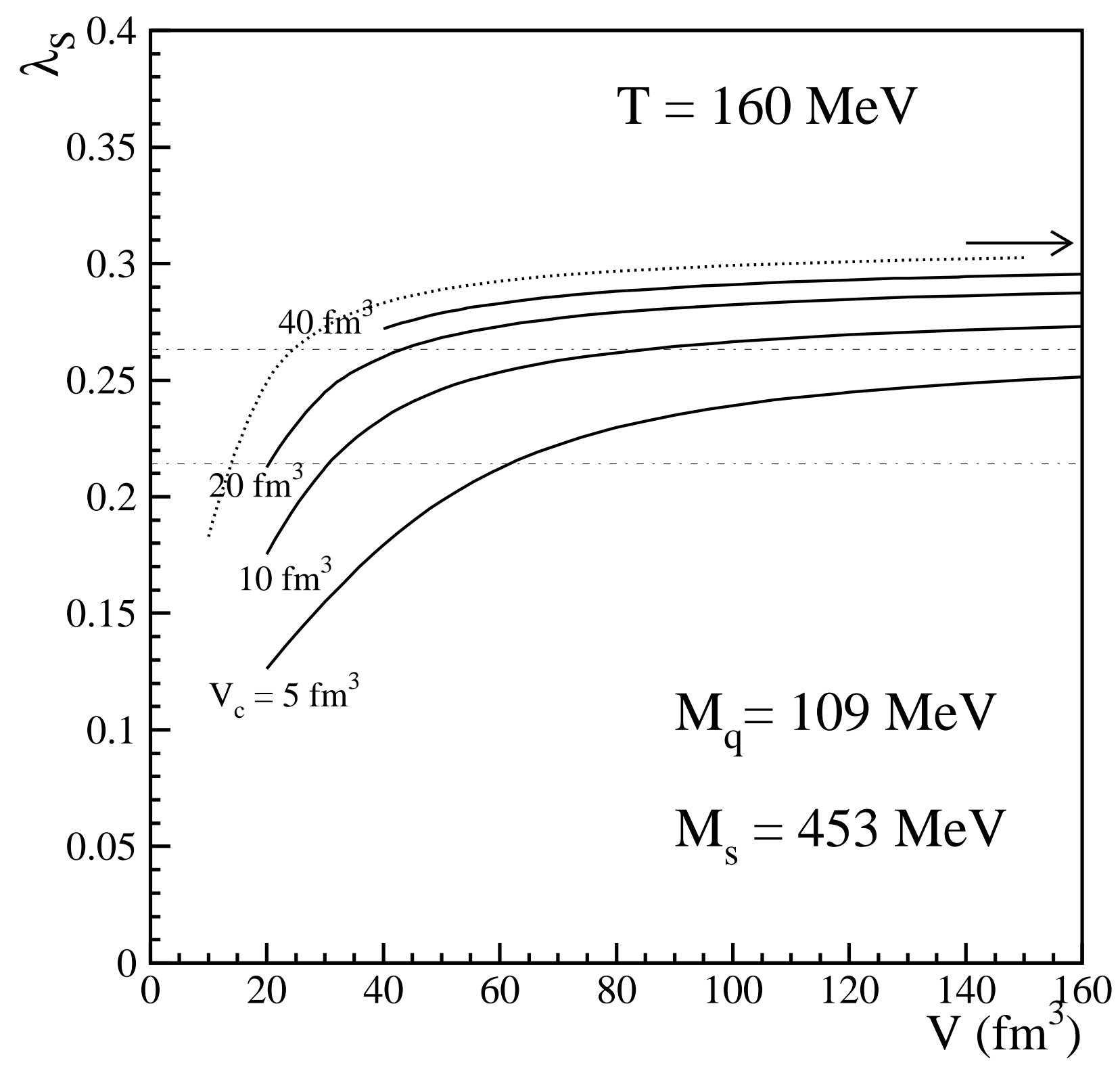

